\title{
Magnitude of spinal muscle damage is not statistically associated with exercise-induced low back pain intensity
}

\author{
Mark D. Bishop, PT, PhDa,b, ${ }^{\star}$, Maggie E. Horn, PTa, Donovan J. Lott, PT, PhDa, Ishu Arpan, \\ $\mathbf{P T}^{\mathrm{a}}$, and Steven Z. George, PT, PhD ${ }^{\mathrm{a}, \mathrm{b}}$ \\ aDepartment of Physical Therapy, University of Florida, PO Box 100154, Gainesville, FL \\ 32610-0154, USA \\ ${ }^{\mathrm{b}}$ Center for Pain Research and Behavioral Health, University of Florida, PO Box 100165, \\ Gainesville, FL 32610-0154, USA
}

\begin{abstract}
BACKGROUND CONTEXT_Findings on imaging of noncontractile anatomic abnormalities and the intensity of low back pain have weak associations because of false-positive rates among asymptomatic individuals. This association might be stronger for contractile tissues.
\end{abstract}

PURPOSE-The purpose of this study was to examine the relationship between location and reports of pain intensity in the low back and exercise-induced muscle damage to the lumbar paraspinal muscles.

STUDY DESIGN—Nondiagnostic observational study in a laboratory setting.

METHODS-Delayed onset muscle soreness was induced in the low back of healthy pain-free volunteers. Measures of pain intensity (100-mm visual analog scale [VAS]) and location (area on the pain diagram) were taken before and 48 hours after exercise. Muscle damage was quantified using mechanical pain thresholds, motor performance deficits, and transverse relaxation time (T2)-weighted magnetic resonance imaging (MRI). Changes pre- to postexercise in signal intensity on T2-weighted imaging within the erector spinae, pain intensity, pain area, mechanical pain threshold, and isometric torque were assessed using paired $t$ tests. Bivariate correlations were conducted to assess associations among muscle damage, pain intensity, and pain drawing area.

RESULTS-Twenty participants volunteered (11 women; average age, 22.3 years; average body mass index, 23.5) for study participation. Reports of pain intensity at 48 hours ranged from 0 to 59 $\mathrm{mm}$ on the VAS. Muscle damage was confirmed by reductions in mechanical threshold $(\mathrm{p}=.011)$ and motor performance $(\mathrm{p}<.001)$ and by changes in T2-weighted MRI $(\mathrm{p}=.007)$. This study was powered to find an association of at least $r=0.5$ to be statistically significant. Correlations of continuous variables revealed no significant correlations between pain intensity and measures of muscle damage (ranging between -0.075 and 0.151 ). There was a significant association between the remaining torque deficit at 48 hours and pain area.

CONCLUSIONS-The results of this study indicate that there was no association between the magnitude of muscle damage in the lumbar erector spinae and reported pain intensity in the low

(C) 2011 Elsevier Inc. All rights reserved.

*Corresponding author. Department of Physical Therapy, University of Florida, PO Box 100154, Gainesville, FL 32610-0154, USA. Tel.: (352) 2736112; fax: (352) 2736109. bish@ufl.edu (M.D. Bishop).

FDA device/drug status: Not applicable.

Author disclosures: $\boldsymbol{M D B}$ : Nothing to disclose. $\boldsymbol{M E H}$ : Nothing to disclose. $\boldsymbol{D J} \boldsymbol{L}$ : Nothing to disclose. $\boldsymbol{I A}$ : Nothing to disclose. $\boldsymbol{S Z G}$ : Nothing to disclose. 
back. In future studies, larger cohorts may report statistically significant associations, but our data suggest that there will be low magnitude potentially indicating limited clinical relevance.

\section{Keywords}

Spinal disorders; Imaging; Referred pain

\section{Introduction}

The search for a pathoanatomic source of a patient's low back pain (LBP) has been difficult, resulting in many authors suggesting that the identity of that source of pain is resistant to the interview and examination process [1-7]. Clinical literature suggests that subjective reports of pain are incongruent with lumbar imaging findings. That is, the determination of a pathoanatomic origin of LBP is obscured by the rate of false-positive findings from imaging studies of the noncontractile elements of the spinal column, such as disc herniations $[8,9]$.

Although these studies have focused on noncontractile structures as a source of LBP, there is little research investigating contractile structures as a source of LBP. Given the lack of support for noncontractile structures as anatomic source of LBP, it would be reasonable to suggest that muscle could be a source of LBP [10-12]. For example, in a study done by Kellgren [10], lumbar paraspinals were sources of back and buttock pain when injected with saline. Therefore, the link between complaints of pain and muscle injury might be stronger than that to noncontractile structures. However, further studies involving clinically relevant models of muscle damage are warranted before such an approach could be advocated in clinical settings.

Over the last several years, magnetic resonance imaging (MRI) techniques have developed to become one of the best methods for visualizing anatomic and structural features of skeletal muscle [13]. Advances in imaging technology are improving the resolution of images produced and consequently the ability to identify anatomic lesions in contractile structures. Transverse relaxation time (T2)-weighted MRI measures have been shown to be sensitive to injury of skeletal muscle and used in both animal and human models to assess muscle damage noninvasively [14-17]. Specific to the purposes of the present study, T2weighted MRI has been used to assess muscle damage after eccentric exercise. Transverse relaxation time is a biophysical property inherent to a tissue and a major source of tissue contrast [18]. Previous studies $[19,20]$ have reported increase in the signal intensities on MRI (or elevated T2) of muscles after eccentric exercise, which can be attributed to an increase in intracellular- or extracellular-free water (edema) resulting from damage induced by such an activity [21,22]. The increase in signal intensity on MRI has also been shown to correlate with the ultrastructural injury to muscle fibers as represented by a reduction in normal $\mathrm{z}$ lines after 48 hours of downhill running on a treadmill [23]. Thus, using change in $\mathrm{T} 2$ could be an effective noninvasive indicator of lumbar muscle damage.

One common form of muscle pain that is associated with muscle damage is delayed onset muscle soreness (DOMS) from novel exercise. Delayed onset muscle soreness is muscle soreness characterized by pain, discomfort, and often acute disability that occurs between 24 and 72 hours after exercise and most often peaks at 48 hours [24,25].

The ability to control the mechanism of muscle injury and perform a noninvasive imaging measure of that injury provides the opportunity to address the efficacy of identification of muscle damage as pathoanatomic pain generators.

Consequently, our purpose was to examine the relationship between location and reports of pain intensity in the low back and contractile sources of pain after exercise-induced muscle 
damage to the lumbar paraspinal muscles. We hypothesized that the intensity of reported pain would be associated with the magnitude of muscle damage in the lumbar musculature. This study will complement the existing literature by being one of the few we are aware of that determines whether contractile tissues have the potential to be more reliable pain generators in comparison with noncontractile elements as LBP generators.

\section{Materials and methods}

Participants were recruited from the university student pool and local community. All participants read and signed an informed consent form approved by the University Institutional Review Board. Participants were excluded if they met any of the following criteria: previous participation in a conditioning program specific to trunk extensors; any current back pain; any chronic medical conditions that may affect pain perception (eg, diabetes, high blood pressure, fibromyalgia, and headaches); kidney dysfunction; muscle damage; major psychiatric disorder; history of previous injury, including surgery to the lumbar spine, renal malfunction, cardiac condition, osteoporosis, or liver dysfunction; and performance of any intervention for symptoms induced by exercise and before the termination of their participation or the protocol.

\section{Procedures}

Participants came to four testing sessions. At the first session, participants completed the informed consent process and a demographic survey, including a series of questions regarding age, height, weight, and exercise habits. Additionally, measures of pain were taken and the baseline MRI. Participants then completed the exercise protocol. Subsequent sessions included measures of pain intensity and measurement of isometric torque of the trunk muscles. Magnetic resonance imaging data were collected at the testing session 48 hours after exercise.

\section{Measures}

Current pain intensity in the low back-Pain intensity in the back after exercise was measured using a self-report of 100-mm visual analog scale (VAS) anchored at one end with "none" and at the other with "worst pain imaginable." Subjects rated their pain intensity by placing a mark along the line. A previous study has indicated that the VAS is a valid ratio measure for pain intensity [26].

A pain diagram was used by participants to indicate the area of the body in which the pain was felt. Participants were instructed to shade the areas on a drawing of a human body in which they felt their pain. The area of the pain was calculated in square millimeters.

Tenderness in paraspinal muscles-Tenderness in paraspinal muscle tissue was assessed using a Microfet 2 hand-held dynamometer (Hoggan Health Industries, Inc., West Jordan, UT, USA). The tip of the dynamometer is equipped with a rubber footplate of $1-\mathrm{cm}$ diameter. During testing, each subject was positioned in prone, and pressure was applied until the subject reported that the sensation changed from pressure to pain. This point is the pressure pain threshold (PPT). At that point, the subject rated pain using a numeric rating scale anchored at 0 (no pain sensation at all) and 100 (worst pain imaginable), and the applied force was recorded in kilogram force. Threshold measures were evoked in the paraspinal muscles bilaterally $2.5 \mathrm{~cm}$ from the spinous processes of L1, L5, and S2 for a total of six ratings. These were averaged to provide a single measure of muscle tenderness.

Magnetic resonance imaging-Magnetic resonance imaging was performed with a Philips Achieva 3.0T MR scanner (Philips Healthcare, Andover, MA, USA) at baseline 
(before any exercise) and 48 hours after the exercise protocol (described in the following sections). The subjects were positioned in a supine headfirst position with the lower legs supported with a foam wedge. The low back was imaged using a three-element phased array (sensitivity encoding) body surface coil receiver. A survey scan was performed from the midthoracic region to the midsacral level. Field of view (anterior-posterior $=200-260 \mathrm{~mm}$, right-left=175-230 mm, and foot-head=103 mm) was then set to collect data from 13 to 14 transaxial slices (thickness $=7 \mathrm{~mm}$; distance between the slices $=8 \mathrm{~mm}$ ) of T2-weighted images that were acquired via a spin echo sequence at five echo times $(30,60,90,120$, and $150 \mathrm{~ms}$ ) and at a repetition time of $3,000 \mathrm{~ms}$.

\section{Exercise protocol}

Before exercise, all subjects completed a warm-up session consisting of riding the stationary bicycle at a speed of 50 to 60 RPM and $1 \mathrm{kp}$ of resistance and 1 minute of static passive stretching of the quadriceps, hamstrings, and gluteal muscles of lower extremities, and the long erector spinae of the trunk. Each subject performed an isometric test of total torque through their available trunk flexion range of motion (ROM) using a MedX lumbar extension exercise machine following the standardized protocol. Subjects were seated in the MedX machine, and the stabilizing straps were attached across the pelvis and knees. The subject was moved through the ROM of the machine in lumbar flexion and extension to determine their available range. The device was locked into place in maximal flexion, and the subject instructed to build up force gradually against a pad in contact with the lower thoracic and upper lumbar spine. Once peak effort was achieved, the subject was instructed to relax, the device released, and the subject returned to an upright position for at least 10 seconds. Isometric testing was administered from the subjects' maximal trunk flexion and every $12^{\circ}$ till maximal trunk extension within their available range.

After baseline torque was recorded from the isometric test, subjects performed bouts of dynamic exercise to the point of volitional fatigue. To perform the dynamic fatiguing exercise, the subjects were seated and restrained in a MedX lumbar extension exercise machine. Subjects performed as many repetitions as possible using a weight load equal to approximately $80 \%$ of the isometric peak torque measured during the isometric test. Each repetition was performed through the full available ROM. Repetitions of both exercises continued until the patient reported being unable to move through a full ROM (volitional fatigue). At the end of the exercise set, the isometric torque test was immediately performed again. Subjects repeated the sequence of dynamic exercise and static testing until total measured torque decreased to approximately $50 \%$ of the baseline measurement. Subjects were instructed not to initiate any pain-relieving medication or apply any palliative intervention to the lumbar spine. At 48 hours after the fatiguing exercise protocol, subjects were asked to perform another isometric test to assess muscle recovery using the same protocol (ie, warm-up followed by testing).

\section{Analysis}

Determining muscle damage-We used a series of metrics to estimate muscle damage and changes in performance. Transverse relaxation time-weighted MRI was the primary indicator of muscle damage. During data processing, the multifidus and paraspinal muscles were identified on the images. The slice corresponding to the L3-L4 intervertebral level was identified based on which slice demonstrated the greatest area of the intervertebral disc at this level. Regions of interest were manually traced for both of these muscles on the subject's right side of the T2-weighted MR images at the single slice for the L3-L4 intervertebral level using OsiriX, an open-source software (OsiriX Imaging software, available from http://www.osirix-viewer.com). Signal intensities were determined for these regions of interest and were plotted against their corresponding echo times. The resultant 
decay curve was then fit with a software program (Origin; Micro-cal Software,

Northampton, MA, USA) using a monoexponential decay algorithm to calculate mean T2 values for both muscles $[14,27]$. These calculations were done to determine mean $\mathrm{T} 2$ values for the multifidus and paraspinal muscles for both time points (baseline and 48 hours) for each subject. The percent difference in $\mathrm{T} 2$ from 48 hours postexercise to baseline was calculated to provide a proxy measure of muscle damage.

In addition, we used PPT to assess peripheral hyperalgesia. We also examined motor performance by assessing isometric trunk extensor torque production. This measure was done after the MRI at 48 hours to avoid any effects of the second torque assessment on muscle hydration. The amount of strength loss is a key component in determining muscle damage. Warren et al. [28] suggested that prolonged strength loss after eccentric exercise is considered to be one of the most valid and reliable indirect measures of muscle damage in humans. Subject's baseline torque production was compared with their isometric torque production at 48 hours to provide an estimate of torque lost across testing sessions. The percent loss of torque during the exercise protocol was also calculated, and participants grouped based on the magnitude of torque loss $50 \%$ fatigue and $51 \%$ or greater.

Statistical analyses-Descriptive statistics were generated for gender and body mass index (BMI). Changes pre- to postexercise in signal intensity on T2-weighted imaging within the erector spinae, PPT, and isometric torque were assessed using Wilcoxon rank sums. Friedman test was used to examine changes in pain intensity over time. Bivariate correlations (Spearman rho) were conducted to assess the possible associations among muscle damage (using change in T2-weighted imaging, PPT, and percent fatigue), pain intensity, and pain drawing area. Our primary interest was association at 48 hours; however, pain intensity at each session was included for analysis. All statistical analyses were conducted using the Statistical Package for the Social Sciences, version 16.0 for Windows (SPSS, Inc., Chicago, IL, USA). Significance level for all tests was set at an alpha level of 0.05 .

\section{Results}

Twenty participants volunteered (11 women; average age, 22.3 years; average BMI, 23.5) for study participation. The participants had an average of 14 years of education and in the majority indicated themselves to be Caucasian (Table 1).

\section{Determining muscle damage}

At 48 hours after exercise, there was an indication of muscle damage by significant increase in signal intensity on T2-weighted imaging in both the short (multifidus) and long erector spinae muscles ( $\mathrm{p}=.007$ and .026 , respectively), indicating increased water content within the muscle boundaries [21,22]. Additionally, changes in contrast in the multifidii and erector spinae muscles were significantly correlated to each other $(\mathrm{r}=0.686, \mathrm{p}=.004)$, indicating similar levels of damage in these muscle groups.

Both PPT and isometric torque were significantly reduced at 48 hours compared with baseline ( $\mathrm{p}=.011$ and <.001; see Fig. 1). In addition, eight participants experienced greater than $51 \%$ loss of torque during the DOMS induction protocol (61\% average loss in torque). These eight participants had significantly greater change in T2 MRI of the multifidii ( $\mathrm{p}=$. 020). An example of changes in MRI is indicated in Fig. 2. Associations among muscle damage measures are shown in Table 2 . 


\section{Pain responses of participants}

Pain intensity ranged from 0 to $50 \mathrm{~mm}$ on the 100 -mm VAS (median, 10) at 24 hours. This was statistically different from baseline $(\mathrm{p}<.001)$. Only two participants did not report pain at 24 hours. The area of pain was 0 to $800 \mathrm{~mm}^{2}$. Pain intensity reported at 48 hours ranged from 0 to 59 on the VAS (median, 10) and was statistically different from baseline ( $\mathrm{p}<.001)$. Four participants reported a pain intensity of 0 at 48 hours. Pain area at 48 hours was increased from baseline $(\mathrm{p}=.002)$ and ranged from 0 to $846 \mathrm{~mm}^{2}$. At 96 hours, 15 participants reported no pain. The remainder ranged from 5 to $36 \mathrm{~mm}$. These reports were not more than baseline but were significantly less than those at 24 and 48 hours.

See Fig. 3 for examples of pain diagrams completed by two participants at 48 hours. There was no association between reported pain intensity and pain area at any time point.

\section{Association among measures}

Correlation coefficients for the correlations between pain intensity and imaging ranged from -0.141 to 0.328 for individual muscles and -0.075 to 0.151 for the average change in T2 intensity (see Table 3 ). None of these correlations were significantly different from zero (p $>.05)$.

There were no significant associations between pain intensity and demographic variables, such as age $(\mathrm{R}=-0.397, \mathrm{p}=.10)$ or BMI $(\mathrm{R}=-0.084, \mathrm{p}=.785)$. There was, however, a significant association between the remaining torque deficit at 48 hours and the area over which the participant experienced pain (indicated by the area shaded in the pain drawing). Point-biserial correlations indicated no differences in changes in $\mathrm{T} 2$ contrast based on sex $(\mathrm{r}=-0.155, \mathrm{p}=.526)$.

\section{Discussion}

The primary finding of this study is that exercise-induced muscle damage to the paraspinals caused LBP in an anatomically appropriate area, but there were no associations among reported pain intensity and amount of muscle damage. Prior work has reported changes in muscle performance [28] and tenderness in the muscle [25,29,30] during DOMS suggesting that these are markers of muscle damage. We confirmed these findings of decreased PPTs in paraspinal muscles and reductions in muscular performance at 48 hours in our model. Participants in our study had significant deficits in PPT, and isometric strength remained reduced (approximately $20 \%$ on average) at 48 hours after exercise. The present study extended prior imaging work by adding a noninvasive metric of muscle damage. Using this, we identified significant changes related to markers of muscle damage. Combined, these findings make us confident that we were successful in causing muscle damage with our exercise protocol and that the lumbar musculature was the peripheral source of pain in these subjects.

The clinical relevance of this exercise-induced pain model is that it caused pain in the lumbar region from contractile elements. The area of the pain experienced by participants in our study was similar to referral areas identified in other studies of pain referral from lumbar musculature $[10,12]$. The area was also very similar to pain referrals noted from zygapophyseal joints of L4-L5 and L5-S1 [31], suggesting that pain referral pattern may identify the general anatomic region of the source of pain, but it is not sufficient to identify the specific anatomic structure as a source of LBP.

These findings did not support our hypothesis that muscle damage and pain intensity would be related as the associations were essentially zero. Although this was contrary to our hypothesis, the findings support previous work indicating weak associations between 
anatomy and reports of intensity of LBP. Prior work examining the links between findings on imaging and pain intensity in LBP has been heavily focused on noncontractile elements of the spinal column. These studies $[8,9,32,33]$ have consistently showed the association between pathoanatomy and pain intensity to be tenuous at best.

Abnormalities of the intervertebral disc, for example, are quite common in people without complaints of LBP. Between 52\% [8] and 81\% [9] of asymptomatic people have evidence of a bulging disc, and 56\% have radial annular tears [9]. Twenty-seven percent of these people show disc protrusion, and $14 \%$ have evidence of disruption of the outer annulus [8]. Some $13 \%$ [32,33] to 19\% [8] of asymptomatic people have evidence of Schmorl's nodes, and osteophytes are present in $47 \%$ of individuals without the symptoms of back pain [33]. The prevalence of all these conditions increases with age; specifically, disc degeneration, facet joint osteoarthritis, or osteophytes is present in more than $90 \%$ of subjects aged older than 64 years [34]. Thus, the relationship between complaints of LBP and imaging findings must be considered cautiously when searching for an anatomic cause of LBP. Our data add to this literature by suggesting that contractile elements are not a likely source for the cause of variability in pain intensity reports from LBP.

If LBP intensity is not related to severity of damage from peripheral contractile elements, then other factors must be considered. Ratings of pain intensity are the result of a complex neurological process during which peripheral input must be interpreted centrally. Peripheral stimuli are relayed along afferent fibers of sensory nerves (myelinated A-delta fibers or unmyelinated nerves $\mathrm{C}$ fibers). These fibers end in the dorsal horn of the spinal cord, where they form synapses with nociception-relay neurons, which in turn form synapses with ascending neurons. Multiple supraspinal sites receive input from ascending neurons reflecting sensory-discriminative components of pain, and behavioral, affective, and emotional responses to pain [35]. Consequently, although all the participants in our study experienced a similar mechanism of injury, the interpretation of that input into ratings of pain intensity varied enough for there to be no relationship between muscle damage and pain intensity in our study.

These results must be interpreted within the context that the participants did not have LBP before participation and, consequently, may be different from patients who seek intervention for their pain. In addition, this induced model of LBP neither does share characteristics with chronic LBP nor was it intended to. Therefore, these results are best generalized to acute LBP. Another limitation was that we did not perform imaging of the contractile elements so these were not measured directly. This limitation is mediated somewhat by our use of younger subjects as these subjects were much more likely to have "normal" imaging findings for lumbar anatomy.

However, the strengths of our model included standardization of damage protocol and control over measures that would otherwise be highly variable. All participants experienced the same mechanism of injury and were measured on standardized physical measures throughout their episode of pain. Also, we are confident that muscular changes occurred in response to the DOMS protocol, and we used sophisticated imaging techniques to quantify this damage although we did not directly measure inflammatory markers. Previous literature indicates correlations between the level of proinflammatory cytokines, such as interleukin-6 [36] or tumor necrosing factor in muscle after exercise [37], and muscle pain. Another benefit of inducing DOMS in healthy participants is frequency of potentially confounding pathological processes in the spine were likely low allowing us to draw conclusions specifically about the associations between muscle damage and pain intensity. 
Lastly, we tested 20 participants in this study. This number of participants makes it possible to identify significant correlations of approximately 0.5 . A correlation of this magnitude is associated with an explanation of about $25 \%$ of the unadjusted variance in the measures. Post hoc analyses indicated that a sample of 70 participants would have resulted in a statistical association between pain intensity at 96 hours and findings on MRI at 48 hours. Even so the expected association was low ( $\mathrm{r}=0.33)$, meaning an explanation of only about 9\% variance in pain intensity ratings. Although such a finding would have been statistically significant with 70 subjects, it would be unlikely to have clinical relevance because of the small amount of variance explained in pain intensity ratings.

In summary, the results of this study indicate that there was no association between the magnitude of muscle damage in the lumbar erector spinae and reported pain intensity in the low back. This finding adds to prior work that focused heavily on noncontractile elements by suggesting that the link between damaged contractile elements identified using imaging and the intensity of LBP is weak.

\section{Acknowledgments}

This project was supported by funding from National Institute of Arthritis and Musculoskeletal and Skin Diseases (K01 AR054331-MDB, MEH), National Center for Medical Rehabilitation Research (K12 HD055929—MDB, DJL), and the Jack Wessel Excellence Award, University of Florida (SZG).

\section{References}

1. Binkley J, Stratford PW, Gill C. Interrater reliability of lumbar accessory motion mobility testing. Phys Ther. 1995; 75:786-92. discussion 793-5. [PubMed: 7659738]

2. Schwarzer AC, Aprill CN, Derby R, et al. Clinical features of patients with pain stemming from the lumbar zygapophysial joints. Is the lumbar facet syndrome a clinical entity? Spine. 1994; 19:11327. [PubMed: 8059268]

3. Spitzer WO, Skovron ML, Salmi LR, et al. Scientific monograph of the Quebec Task Force on Whiplash-Associated Disorders: redefining "whiplash" and its management. Spine. 1995; 20:1S73S. [PubMed: 7604354]

4. Maher C, Adams R. Reliability of pain and stiffness assessments in clinical manual lumbar spine examination. Phys Ther. 1994; 74:801-9. discussion 809-11. [PubMed: 8066107]

5. Maigne JY, Aivaliklis A, Pfefer F. Results of sacroiliac joint double block and value of sacroiliac pain provocation tests in 54 patients with low back pain. Spine. 1996; 21:1889-92. [PubMed: 8875721]

6. Dreyfuss P, Michaelsen M, Pauza K, et al. The value of medical history and physical examination in diagnosing sacroiliac joint pain. Spine. 1996; 21:2594-602. [PubMed: 8961447]

7. Waddell G. Low back pain: a twentieth century health care enigma. Spine. 1996; 21:2820-5. [PubMed: 9112705]

8. Jensen MC, Brant-Zawadzki MN, Obuchowski N, et al. Magnetic resonance imaging of the lumbar spine in people without back pain. N Engl J Med. 1994; 331:69-73. [PubMed: 8208267]

9. Stadnik TW, Lee RR, Coen HL, et al. Annular tears and disk herniation: prevalence and contrast enhancement on MR images in the absence of low back pain or sciatica. Radiology. 1998; 206:4955. [PubMed: 9423651]

10. Kellgren JH. Observations on referred pain arising from muscle. Clin Sci. 1938; 3:175-90.

11. Kellgren JH. On the distribution of pain arising from deep somatic structures with charts of the segmental areas. Clin Sci. 1939; 4:35-46.

12. Kuslich SD, Ulstrom CL, Michael CJ. The tissue origin of low back pain and sciatica: a report of pain response to tissue stimulation during operations on the lumbar spine using local anesthesia. Orthop Clin North Am. 1991; 22:181-7. [PubMed: 1826546]

13. Patten C, Meyer RA, Fleckenstein JL. T2 mapping of muscle. Semin Musculoskelet Radiol. 2003; 7:297-305. [PubMed: 14735428] 
14. Frimel TN, Walter GA, Gibbs JD, et al. Noninvasive monitoring of muscle damage during reloading following limb disuse. Muscle Nerve. 2005; 32:605-11. [PubMed: 16003743]

15. LeBlanc AD, Jaweed M, Evans H. Evaluation of muscle injury using magnetic resonance imaging. Clin J Sport Med. 1993; 3:26-30. [PubMed: 11538201]

16. Sorichter S, Koller A, Haid C, et al. Light concentric exercise and heavy eccentric muscle loading: effects on CK, MRI and markers of inflammation. Int J Sports Med. 1995; 16:288-92. [PubMed: 7558524]

17. Liu M, Bose P, Walter GA, et al. Changes in muscle T2 relaxation properties following spinal cord injury and locomotor training. Eur J Appl Physiol. 2006; 97:355-61. [PubMed: 16770473]

18. Hashemi, RH.; Bradley, WG.; Lisanti, CJ. MRI The basics. Philadelphia, PA: Lippincott Williams \& Wilkins; 2004.

19. Foley JM, Jayaraman RC, Prior BM, Pivarnik JM. MR measurements of muscle damage and adaptation after eccentric exercise. J Appl Physiol. 1999; 87:2311-8. [PubMed: 10601183]

20. Nosaka K, Clarkson PM. Changes in indicators of inflammation after eccentric exercise of the elbow flexors. Med Sci Sports Exerc. 1996; 28:953-61. [PubMed: 8871903]

21. Ploutz-Snyder LL, Convertino VA, Dudley GA. Resistance exercise-induced fluid shifts: change in active muscle size and plasma volume. Am J Physiol. 1995; 269:R536-43. [PubMed: 7573553]

22. Ploutz-Snyder LL, Nyren S, Cooper TG, et al. Different effects of exercise and edema on T2 relaxation in skeletal muscle. Magn Reson Med. 1997; 37:676-82. [PubMed: 9126941]

23. Nurenberg P, Giddings CJ, Stray-Gundersen J, et al. MR imaging-guided muscle biopsy for correlation of increased signal intensity with ultrastructural change and delayed-onset muscle soreness after exercise. Radiology. 1992; 184:865-9. [PubMed: 1509081]

24. Armstrong RB. Mechanisms of exercise-induced delayed onset muscular soreness: a brief review. Med Sci Sports Exerc. 1984; 16:529-38. [PubMed: 6392811]

25. George SZ, Dover GC, Fillingim RB. Fear of pain influences outcomes after exercise-induced delayed onset muscle soreness at the shoulder. Clin J Pain. 2007; 23:76-84. [PubMed: 17277648]

26. Price DD, McGrath PA, Rafii A, Buckingham B. The validation of visual analogue scales as ratio scale measures for chronic and experimental pain. Pain. 1983; 17:45-56. [PubMed: 6226917]

27. Fisher MJ, Meyer RA, Adams GR, et al. Direct relationship between proton T2 and exercise intensity in skeletal muscle MR images. Invest Radiol. 1990; 25:480-5. [PubMed: 2345077]

28. Warren GL, Lowe DA, Armstrong RB. Measurement tools used in the study of eccentric contraction-induced injury. Sports Med. 1999; 27:43-59. [PubMed: 10028132]

29. Nie H, Arendt-Nielsen L, Madeleine P, Graven-Nielsen T. Enhanced temporal summation of pressure pain in the trapezius muscle after delayed onset muscle soreness. Exp Brain Res. 2006; 170:182-90. [PubMed: 16328284]

30. Nie H, Madeleine P, Arendt-Nielsen L, Graven-Nielsen T. Temporal summation of pressure pain during muscle hyperalgesia evoked by nerve growth factor and eccentric contractions. Eur J Pain. 2008; 13:704-10. [PubMed: 18710817]

31. Marks R. Distribution of pain provoked from lumbar facet joints and related structures during diagnostic spinal infiltration. Pain. 1989; 39:37-40. [PubMed: 2530485]

32. Hitselberger WE, Witten RM. Abnormal myelograms in asymptomatic patients. J Neurosurg. 1968; 28:204-6. [PubMed: 5643913]

33. Torgerson WR, Dotter WE. Comparative roentgenographic study of the asymptomatic and symptomatic lumbar spine. J Bone Joint Surg Am. 1976; 58:850-3. [PubMed: 134040]

34. Videman T, Nurminen M, Troup JD. 1990 Volvo Award in clinical sciences. Lumbar spinal pathology in cadaveric material in relation to history of back pain, occupation, and physical loading. Spine. 1990; 15:728-40. [PubMed: 2146754]

35. Calvino B, Grilo GM. Central pain control. Joint Bone Spine. 2006; 73:10-6. [PubMed: 15922645]

36. MacIntyre DL, Sorichter S, Mair J, et al. Markers of inflammation and myofibrillar proteins following eccentric exercise in humans. Eur J Appl Physiol. 2001; 84:180-6. [PubMed: 11320633]

37. Clarkson PM, Hubal MJ. Exercise-induced muscle damage in humans. Am J Phys Med Rehabil. 2002; 81:S52-69. [PubMed: 12409811] 


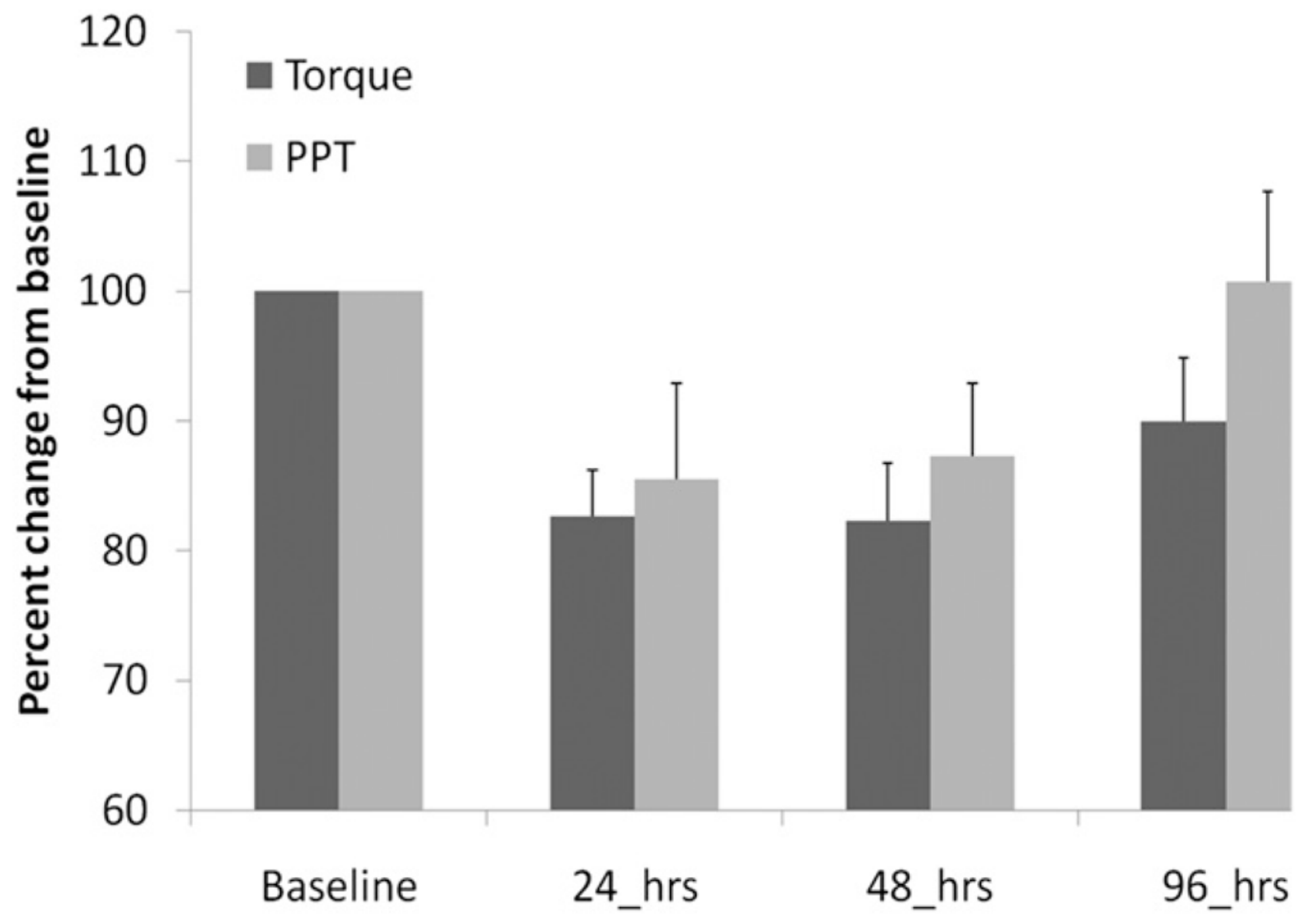

Fig. 1.

Percentage changes in pressure pain threshold (PPT) and total isometric torque (torque). 


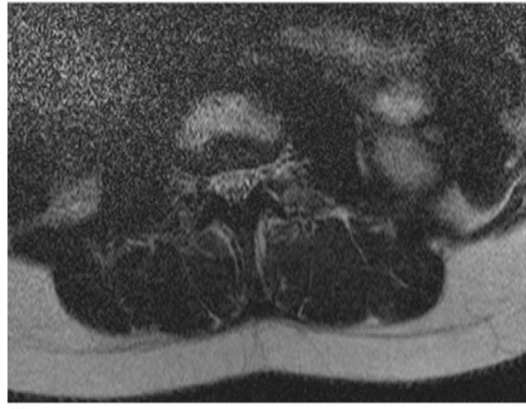

Pre

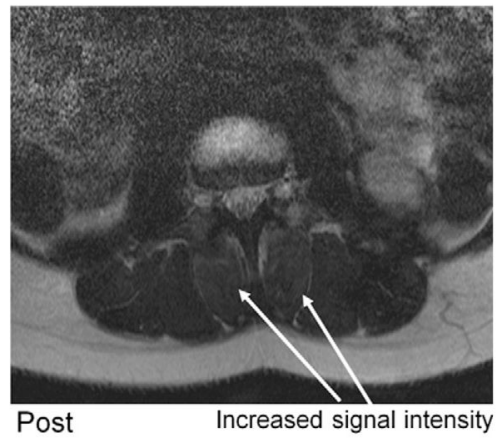

Fig. 2.

T2-weighted magnetic resonance imaging (Left) pre-exercise and (Right) postexercise. Note the increased signal intensity (brightness) at the multifidii 48 hours (Right) after exercise (Left) relative to baseline. 

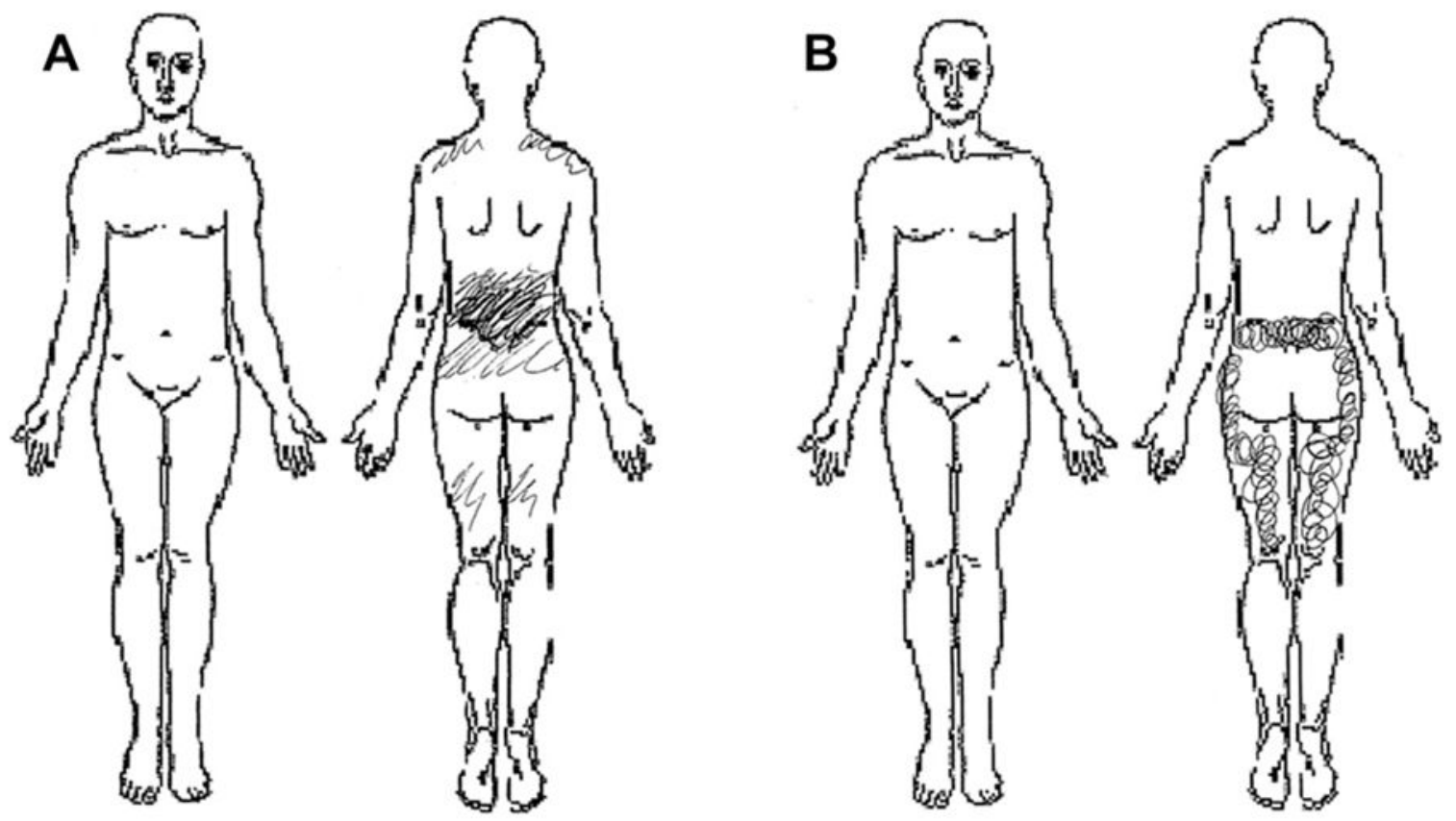

Fig. 3.

Representative pain diagrams. The area of pain is approximately $450 \mathrm{~mm}^{2}$ in both diagrams; however, pain intensity in Participant A was 7, whereas pain intensity in Participant B was 45. 


\section{Table 1}

Demographic characteristics of participants

\begin{tabular}{lcl}
\hline Participant characteristic & Mean & Standard deviation \\
\hline Age (y) & 22.3 & 3.4 \\
BMI $\left(\mathrm{km}^{-2}\right)$ & 23.5 & 5.0 \\
Education (y) & 14 & 2 \\
Gender (number of females) & 11 & $55 \%$ \\
Ethnicity & & \\
Caucasian & 17 & \\
$\quad$ African American & 2 & \\
Asian & 1 \\
Native American & - \\
Pacific Islander & - \\
\hline
\end{tabular}

BMI, body mass index. 
Table 2

Association among measures of muscle damage

\begin{tabular}{lcccc}
\hline Measure of muscle damage & T2 change-erector spinae & T2 change-average & Torque loss at 48 h & PPT change \\
\hline T2 change-multifidii & $0.686^{*}$ & $0.914^{*}$ & 0.225 & -0.196 \\
T2 change-erector spinae & $0.887^{*}$ & 0.211 & 0.169 \\
T2 change-average & & 0.250 & -0.017 \\
Torque loss at $48 \mathrm{~h}$ & & -0.229 \\
\hline
\end{tabular}

PPT, pressure pain threshold.

Torque loss is the deficit remaining at 48 hours from the baseline isometric torque test expressed as a percentage of baseline torque. Significant association $\mathrm{p}<.01$. 
